\section{The distinction between personality disorder and mental illness}

\author{
R. E. KENDELL
}

\begin{abstract}
Background Proposals by the UK Government for preventive detention of people with 'dangerous severe personality disorders' highlight the unresolved issue of whether personality disorders should be regarded as mental illnesses.
\end{abstract}

\begin{abstract}
Aims To clarify the issue by examining the concepts of psychopathy and personality disorder, the attitudes of contemporary British psychiatrists to personality disorders, and the meaning of the terms 'mental illness' and 'mental disorder'.
\end{abstract}

Method The literature on personality
disorder is assessed in the context of four
contrasting concepts of illness or disease.

Results Whichever of the four concepts or definitions is chosen, it is impossible to conclude with confidence that personality disorders are, or are not, mental illnesses; there are ambiguities in the definitions and basic information about personality disorders is lacking.

Conclusions The historical reasons for regarding personality disorders as fundamentally different from mental illnesses are being undermined by both clinical and genetic evidence. Effective treatments for personality disorders would probably have a decisive influence on psychiatrists' attitudes.

Declaration of interest None.

\section{BACKGROUND}

\section{The legislative background}

Psychiatrists, and perhaps British psychiatrists more than most, are ambivalent about whether to regard personality disorders as mental illnesses. Until recently, there was no compelling reason for attempting to resolve the issue, but the situation was transformed in 1999 when the UK Government made it clear that it intended to introduce legislation in England and Wales for the compulsory and potentially indefinite detention of people with what it called 'dangerous severe personality disorder', whether or not they had been convicted of a serious criminal offence (Home Office \& Department of Health, 1999). It is likely that some of these people, almost all of them men, will be detained in prisons and others in high-security hospitals. However, the European Convention on Human Rights, which was incorporated into UK legislation by the Human Rights Act 1998, prohibits the detention of anyone who has not been convicted by a competent court unless they are 'of unsound mind, alcoholics or drug addicts or vagrants' or their detention is 'for the prevention of the spreading of infectious diseases'. This means that, to prevent a successful judicial challenge, the Government will have to argue that the potentially dangerous men it wishes to incarcerate are 'of unsound mind', and this means maintaining that they have personality disorders, and that personality disorders are mental disorders.

At present English mental health legislation, which dates from 1983 but had its origins in the recommendations of a Royal Commission in the 1950s, distinguishes between mental illness and psychopathic disorder, but the Government intends to abandon the concept of psychopathic disorder and introduce a new 'broad definition of mental disorder covering any disability or disorder of mind or brain' which will cover personality disorders as well as mental illnesses (Department of Health \& Home Office, 2000).

\section{Implications of the term 'personality disorder'}

The term 'psychopathic' was coined by the German psychiatrist Koch in 1891, and he said firmly that 'even in the bad cases the irregularities do not amount to mental disorder' (Lewis, 1974). What Koch meant by mental disorder, however, was largely restricted to insanity and idiocy, and his concept of 'psychopathic inferiorities' embraced most non-psychotic mental illness as well as what we now call personality disorder or psychopathy. Even so, Kurt Schneider subsequently argued that personality disorders are simply 'abnormal varieties of sane psychic life' (Schneider, 1950), and therefore of little concern to psychiatrists, a view that is still influential in Germany today.

Many - perhaps most - contemporary British psychiatrists seem not to regard personality disorders as illnesses. Certainly, it is commonplace for a diagnosis of personality disorder to be used to justify a decision not to admit someone to a psychiatric ward, or even to accept them for treatment - a practice that understandably puzzles and irritates the staff of accident and emergency departments, general practitioners and probation officers, who find themselves left to cope as best they can with extremely difficult, frustrating people without any psychiatric assistance. The reasons for this attitude were explored by Lewis \& Appleby (1988). Using ratings of case vignettes by 240 experienced psychiatrists, they showed that suicide attempts and other behaviours by patients previously diagnosed as having personality disorders were commonly regarded as manipulative and under voluntary control rather than the result of illness, and that the patients themselves were generally regarded as irritating, attention-seeking, difficult to manage and unlikely to comply with advice or treatment.

Personality disorders are described in the International Classification of Mental and Behavioural Disorders (ICD-10) as 'deeply ingrained and enduring behaviour patterns, manifesting themselves as inflexible responses to a broad range of personal and social situations'; they represent 'either extreme or significant deviations from the way the average individual in a 
given culture perceives, thinks, feels, and particularly relates to others' and are 'developmental conditions, which appear in childhood or adolescence and continue into adulthood' (World Health Organization, 1992a). They are distinguished from mental illness by their enduring, potentially lifelong nature and by the assumption that they represent extremes of normal variation rather than a morbid process of some kind. Whether or not these assumptions are justified, there is broad agreement that personality disorders are important to psychiatrists because they impinge on clinical practice in so many different ways. People with personality disorders are at increased risk of several different mental disorders, including depressions and anxiety disorders, suicide and parasuicide, and misuse of and dependence on alcohol and other drugs. In addition, people with schizotypal personalities are at increased risk of schizophrenia and those with anancastic personalities are at increased risk of obsessivecompulsive disorders. The presence of a personality disorder also complicates the treatment of most other mental disorders, most obviously because the individuals concerned do not easily form stable relationships with their therapists or take prescribed medication regularly. Indeed, in group settings they often disrupt the treatment of other patients as well. Finally, with or without treatment, the prognosis of most mental disorders is worsened by coexistent personality disorder. Because of these important, complex relationships, it is taken for granted that psychiatrists need to be alert to the presence of personality disorder, even if, as is often the case, the disorder does not correspond to any of the distinct types described in textbooks and listed in glossaries. The contentious issues are whether personality disorders are amenable to treatment, and whether people displaying these habitual abnormalities of behaviour deserve to be accorded the privileges of the 'invalid role'.

If personality disorders are not to be regarded as mental illnesses despite their undisputed relevance to psychiatric practice, the obvious alternative is to regard them as risk factors and complicating factors for a wide range of mental disorders, in much the same way that obesity is a risk factor for diabetes, myocardial infarction, breast cancer, gallstones and osteoarthritis, and complicates the management of an even wider range of conditions. Like personality disorder, obesity is listed as a disease in the ICD-10: it is coded E66 as an endocrine, nutritional or metabolic disease (World Health Organization, 1992b). Even so, most doctors, whether they be general practitioners, physicians or surgeons, are reluctant to attempt to treat obesity, either because they regard the condition as the result of self-indulgence rather than metabolic abnormality, or simply because they have no effective treatment to offer (Baxter, 2000).

Against the background of the UK Government's legislative proposals it is clearly important for British psychiatrists, legislators and jurists to decide whether personality disorder, or any subset of it, is a mental illness or mental disorder. Unfortunately, there is no agreed medical definition of either term. The World Health Organization has always avoided defining 'disease', 'illness' or 'disorder', and in its current (ICD-10) classification of mental and behavioural disorders (which includes personality disorders) it simply states that 'the term disorder is used throughout the classification, so as to avoid even greater problems inherent in the use of terms such as disease and illness. Disorder is not an exact term, but it is used here to imply the existence of a clinically recognisable set of symptoms or behaviour associated in most cases with distress and with interference with personal functions' (World Health Organization, 1992a). The current edition of the Diagnostic and Statistical Manual (DSM-IV) of the American Psychiatric Association, which likewise includes personality disorders, does contain a detailed definition of the term 'mental disorder', but although this runs to 146 words it is not cast in a way that allows it to be used as a criterion for deciding what is and is not mental disorder (American Psychiatric Association, 1994). It is important to note, though, that DSM-IV does stipulate that 'neither deviant behavior nor conflicts that are primarily between the individual and society are mental disorders unless the deviance or conflict is a symptom of a dysfunction in the individual', and that there is a similar unambiguous statement in ICD-10.

There is no fundamental difference between so-called mental illnesses or disorders and physical illnesses or disorders; both are simply subsets of illness or disorder in general (Kendell, 1993; American Psychiatric Association, 1994). This implies that the basic issue is the meaning of the terms 'illness' or 'disorder' in general. Even in this wider context, however, there is no agreement, and until recently surprisingly little discussion.

\section{DEFINITIONS OF ILLNESS OR DISORDER}

The most contentious issue is whether disease, illness or disorder (like the World Health Organization, I regard these terms as roughly synonymous) are scientific or biomedical terms, or whether they are socio-political terms which necessarily involve a value judgement. Physicians have generally maintained, or simply assumed, that they are biomedical terms, while philosophers and social scientists have generally argued that they are inherently socio-political, but this is not invariable. The American physician Lester King asserted long ago that 'biological science does not try to distinguish between health and disease ... health or disease are value judgements' (King, 1954). Conversely, the philosopher Bourse has argued that 'disease, the theoretical concept ... applies indifferently to organisms of all species. That is because ... it is to be analysed in biological rather than ethical terms' (Bourse, 1975). I myself once argued that disease ought to be a biomedical concept (Kendell, 1975), but subsequently became convinced that value judgements were probably inescapable (Kendell, 1986). The issue has attracted much attention in the USA in the past decade, mainly in response to the publication of a closely argued analysis of the concept of mental disorder by Wakefield (1992).

\section{STATUS OF PERSONALITY DISORDERS}

The question of whether personality disorders are mental disorders cannot usefully be discussed until agreement has been reached on the implications of the term 'mental disorder', and at present there are at least four quite different, rival concepts of disease or disorder, which are summarised below.

\section{Socio-political}

Although it has been suggested in the past that disease is simply what doctors treat (e.g. Kräupl Taylor, 1971), there are no contemporary advocates for such a 
simplistic view. The simplest plausible socio-political definition is that a condition is regarded as a disease if it is agreed to be undesirable (an explicit value judgement) and if it seems on balance that physicians (or health professionals in general) and their technologies are more likely to be able to deal with it effectively than any of the potential alternatives, such as the criminal justice system (treating it as crime), the church (treating it as sin) or social work (treating it as a social problem).

There is general agreement that the personality traits and behaviours characteristic of personality disorders are undesirable, certainly from society's viewpoint and probably from that of most of the individuals concerned as well. It is unclear, though, whether psychiatry or clinical psychology yet possesses effective treatments for most types of personality disorder. There is evidence that borderline personalities can be helped either by dialectical behaviour therapy (Linehan et al, 1991) or by an analytically oriented day hospital regimen (Bateman \& Fonagy, 1999), but little evidence that this is so for any of the other types. Attempts to change the enduring attitudes and behaviours of personality disorders are not often made; when they are, the treatment is often given up prematurely; and few random allocation trials with adequate long-term follow-up of any form of treatment for any type of personality disorder, other than the borderline type, have been conducted. Moreover, in the case of antisocial personality disorders, the most contentious group, it is undoubtedly the case that, worldwide, the majority are 'managed' most of the time by the criminal justice system rather than by health services.

\section{Biomedical}

The most plausible purely biomedical criterion of disease is the biological disadvantage proposed by Scadding (1967). Scadding, a chest physician, defined a disease as 'the sum of the abnormal phenomena displayed by a group of living organisms in association with a specified common characteristic or set of characteristics by which they differ from the norm for the species in such a way as to place them at a biological disadvantage'. $\mathrm{He}$ never explained what he meant by biological disadvantage, but Kendell (1975) and Bourse (1975) both argued that it must at least encompass reduced fertility and life expectancy.

Little is known about the fertility of people with personality disorders, but there is good evidence that their life expectancy is reduced. Martin et al (1985) studied the mortality over 6-12 years of 500 former psychiatric out-patients in St Louis and found that antisocial personality disorder was associated with a greatly increased mortality (standardised mortality ratio $8.57, P=0.01$ ), mainly from suicide, homicide and accidents. Others have investigated the mortality associated with personality disorder as a whole and found it to be raised in both men and women (Harris \& Barraclough, 1998).

\section{Biomedical and socio-political}

Wakefield, who is a philosopher with a background in social work, argues that mental disorders are biological dysfunctions that are also harmful. This implies that the concept of mental disorder inevitably involves both a scientific or biomedical criterion (dysfunction) and an explicit value judgement or socio-political criterion - what Wakefield (1992, 1999) calls harm and the World Health Organization (1980) defines as handicap - and is attractive because it reconciles the sociopolitical and biomedical camps. It also seems to reflect the often intuitive ways in which physicians make disease attributions and does not obviously have unacceptable implications.

Wakefield originally proposed that dysfunction should imply the failure of a biological mechanism to perform a natural function for which it had been designed by evolution, but Lilienfeld \& Marino (1995) subsequently pointed out that this evolutionary perspective raises many problems. Too little is known about the evolution of most of the higher cerebral functions whose malfunctioning presumably underlies most mental disorders; mood states such as anxiety and depression may have evolved as biologically adaptive responses to danger or loss rather than being failures of evolutionarily designed functions; and several important cognitive abilities, such as reading and calculating, have been acquired too recently to be plausibly regarded as natural functions designed by evolution. It is, of course, perfectly possible in principle to define dysfunction without reference to either evolution or biological disadvantage, and the DSM-IV definition of mental disorder states that it must currently be considered a manifestation of a behavioral, psychological or biological dysfunction'. The problem is that current understanding of the cerebral mechanisms underlying basic psychological functions such as perception, abstract reasoning and memory is too limited for it to be possible in most cases to do more than infer the probable presence of a biological dysfunction; and rejecting both the evolutionary (Wakefield) and biological disadvantage (Scadding) criteria could open the way to regarding a wide range of purely social disabilities (such as aggressive, uncooperative behaviour or an inability to resist lighting fires or stealing) as mental disorders.

The evidence that personality disorders are harmful is quite strong and not restricted to clinic populations. Drake \& Vaillant (1985), for example, compared 86 middle-aged men who met DSM-III criteria for personality disorder with 283 men who did not. Both groups had originally been members of a cohort of mainly working-class, non-delinquent adolescent boys previously studied as a control population in Boston by the Gluecks, so extensive background information was available for all 369. Compared with the 283 men without personality disorders, the 86 personality-disordered men (only six of whom had disorders of antisocial type) had poor mental health $(79 \% v$. $14 \%)$, poor occupational performance and job satisfaction, and poor social competence $(58 \% v .10 \%)$, and although alcohol dependence or misuse was partly responsible for their poor occupational performance, it made little contribution to their poor mental health and social competence.

It is much harder to establish that personality disorder involves dysfunction, in the sense of 'failure of a mental mechanism to perform a natural function for which it was designed by evolution' (Wakefield, 1992). Indeed, it has been argued that several of the characteristic features of antisocial personality disorder, such as manipulation, aggression and deception, were originally successful predatory strategies that evolved in a prehistoric social environment (Lilienfeld \& Marino, 1995). Fundamentally, there are two sources of difficulty. The behaviours and attitudes that define personality disorders are probably graded traits present to a lesser degree in many other people, and also quite different in different types of personality 
disorder; and as yet little is known of the underlying mechanisms of which they are a manifestation. It could be argued, for example, that the impulsiveness and liability to become dependent on drugs or alcohol - which are such prominent, and frequently lethal, features of antisocial disorders - are prima facie evidence of an underlying dysfunction, but in the absence of any understanding of the cerebral mechanisms involved the argument remains inconclusive.

\section{Ostensive}

Lilienfeld \& Marino (1995) maintain that mental disorder is an ostensive or Roschian concept, implying that the term can only be understood by considering the prototypes of mental disorder. If this argument is accepted - and it is not easily dismissed it is impossible even in principle to determine whether personality disorders are mental disorders, because mental disorder is inherently indefinable. The only criterion is whether personality disorders are sufficiently similar to the prototypes of mental disorder (schizophrenia and major depression, perhaps), and similarity is obviously open to a range of interpretations. It is important to note, though, that both the World Health Organization and the American Psychiatric Association include personality disorders in their classifications of mental disorders, without explanation or apology, and have always done so, which implies that both bodies do regard them as sufficiently similar to warrant inclusion. The fact that some forensic psychiatrists see close similarities between personality disorders and schizophrenia, both in the extent of the disturbance of personality involved and in their need for treatment (Blackburn et al, 1993), is also relevant.

\section{DISCUSSION}

It seems clear from this analysis that it is impossible at present to decide whether personality disorders are mental disorders or not, and that this will remain so until there is an agreed definition of mental disorder. It is also apparent that personality disorders are conceptually heterogeneous, that information about them is limited, and that existing knowledge is largely derived from unrepresentative clinical populations. The clinical literature on personality disorders - indeed, the basic concept of personality disorder - has few points of contact with the psychological literature on personality structure and development, and little is known of the cerebral mechanisms underlying personality traits. There is also a glaring need for a better classification of personality disorders and for more long-term follow-up studies of representative samples, derived from community rather than clinical populations, to answer basic questions about the extent, nature and time course of the handicaps associated with different types of personality disorder.

\section{Epistemologic arguments}

It could be argued that personality disorders are mental disorders on the grounds that their high mortality clearly constitutes a biological disadvantage, the key criterion of Scadding's concept of disease. However, there is little support for this definition, and (apart from antisocial personalities) the reduction in life expectancy is fairly modest. If Wakefield's definition, giving a central role to dysfunction, comes to be adopted as a general definition of mental disorder the issue may remain unresolved for some time, because it may not be possible to decide whether there is a dysfunction of some natural mechanism until much more is known of the cerebral mechanisms underlying key personality characteristics such as empathy, impulse control and emotional stability. (There is, though, already evidence that low central serotonergic activity may underlie impulsive, aggressive behaviour in a wide range of settings: see Coccaro \& Kavoussi, 1997.)

Although it is difficult to provide irrefutable arguments that personality disorders are mental disorders, it is equally difficult to argue with conviction that they are not. The fact that they have been included in the two most influential and widely used classifications of mental disorders (the ICD and the DSM) for the past half-century is difficult to disregard, whether or not one accepts the view that mental disorder is an ostensive concept. It could be argued, though, that the crucial issue is not whether personality disorder is embraced by any particular definition or concept of mental illness, but what kinds of considerations lead doctors to change their minds about assignations of illness, and in this context two issues loom large.

\section{Assumptions about aetiology and time course}

The first is the validity of the assumptions about aetiology and time course which originally underpinned the distinction between personality disorder and mental illness. The former was assumed to be part of the normal spectrum of personality variation and to be stable throughout adult life; the latter to be the result of a morbid process of some kind and to have a recognisable onset and time course. These assumptions both appear increasingly questionable, and as a result the distinction between illness and personality disorder is starting to break down. Some schizophrenic illnesses have the same time course as a personality disorder: they develop during adolescence and persist relatively unchanged throughout adult life. More significantly, it is becoming increasingly clear that the genetic bases of affective personality disorders and mood disorders, and of schizotypal personality disorder and schizophrenia, have much in common. As a result, the affective personality disorder of ICD-9 has been replaced in ICD-10 by two new mood disorders, cyclothymia and dysthymia, 'because of evidence from family studies that they are genetically related to the mood disorders, and because they are sometimes amenable to the same treatments'. For similar reasons, schizotypal disorderwhich is listed as a personality disorder in DSM-IV - is classified with schizophrenia and delusional disorders (F20-29) in ICD-10 despite the fact that 'its evolution and course are usually those of a personality disorder' (World Health Organization, 1992a). Most disconcertingly of all, avoidant personality disorder has so much in common with the mental illness known as generalised social phobia that it is suspected that 'they may be alternative conceptualisations of the same or similar conditions' (American Psychiatric Association, 1994). Unsurprisingly, such problems are leading many American psychiatrists to question the value of the distinction between Axis I and Axis II disorders in DSM-IV, despite the statement in its manual that the coding of personality disorders on axis II should not be taken to imply that their pathogenesis or range of appropriate treatment is fundamentally different from that for the disorders coded on axis I' (American Psychiatric Association, 1994). 


\section{The influence of effective therapies}

The second issue is the influence on medical attitudes of the acquisition of an apparently effective therapy. For nearly 150 years, claims that alcoholism was a disease, from Thomas Trotter in 1804 to Alcoholics Anonymous in the 1930s and 1940s, cut little ice with the medical profession. It was only in the late 1940s and 1950s, when disulfiram became available, that doctors changed their minds. Now, of course, it is evident that disulfiram is not generally an effective therapy, but in its early years enthusiastic reports of cures were published in many different countries, and it was against this background that the World Health Organization decided to include alcoholism itself, as distinct from alcoholic psychoses and acute alcohol poisoning, in the ICD, and medical organisations throughout the world issued formal statements to the effect that alcoholism was a disease after all. The reasoning involved suggests an acceptance of the socio-political definition described above, although this has rarely commended itself to the medical profession. It does seem, none the less, that possession of an apparently effective treatment can produce a decisive change in medical opinion, and Campbell et al (1979) showed that an established medical role in diagnosis or treatment has more influence on doctors' concepts of disease than on those of the public. At present, neither personality disorder nor obesity is accepted as a genuine illness by most British doctors; but as effective drugs for treating obesity come into widespread use over the next decade it is likely that obesity will come to be accepted as a genuine metabolic disorder, and the same may happen to personality disorders. Indeed, it is already happening to the 'borderline' disorders as evidence accumulates that the disruptive and self-destructive behaviours that characterise the disorder are amenable to forms of psychotherapy (Linehan et al, 1991; Bateman \& Fonagy, 1999). There is also some evidence that fluoxetine reduces irritability and aggression in people with a variety of personality disorders (Coccaro \& Kavoussi, 1997). If, therefore, the psychiatrists and politicians who maintain that 'antisocial personality disorder' has as good a claim to being accepted as a mental disorder as schizophrenia can demonstrate that it responds to some form of treatment that is not simply a disciplined environment, it is likely that the opposition will melt

\section{CLINICAL IMPLICATIONS}

- Because the term mental illness has no agreed meaning it is impossible to decide with confidence whether or not personality disorders are mental illnesses.

- The historical reasons for regarding personality disorders as fundamentally different from illnesses are being undermined by both clinical and genetic evidence.

- The introduction of effective treatments would probably have a decisive influence on psychiatrists' attitudes.

\section{LIMITATIONS}

No comprehensive literature review was carried out.

The literature on personality disorders is not extensive and consists mainly of clinical rather than population-based studies.

- Psychiatrists' attitudes are influenced by the setting in which health care is provided.

R. E. KENDELL, FRSE, University of Edinburgh, 3 West Castle Road, Edinburgh EHI0 5AT, UK

(First received 23 November 2000, final revision II June 200I, accepted I2 June 200I)

away, and the same will be true for other types of personality disorder.

\section{Economic and cultural influences}

Finally, it is necessary to acknowledge the influence of the setting in which psychiatric care is delivered. For the past 50 years the constraints of the National Health Service have ensured that the time and energy of most British psychiatrists have been fully occupied treating patients with severe mental illnesses. As a result, National Health Service psychiatrists have generally been reluctant to add to their workload by also accepting responsibility for people with deeply ingrained maladaptive behaviours for which there were no proven therapies. In North America the situation has been different. The prevailing systems of health care have facilitated the development of both private office practice and various forms of psychotherapy, and the psychoanalytic concept of 'borderline personality disorder' has provided a rationale for treating, as personality disorders, large numbers of patients who in Britain would mostly be regarded as suffering from recurrent depression. These economic and cultural differences have probably contributed to the comparative reluctance of
British psychiatrists to accept personality disorders as mental disorders.

\section{REFERENCES}

American Psychiatric Association (1994) Diagnostic and Statistical Manual of Mental Disorders (4th edn) (DSM-IV).Washington, DC: APA.

Baxter, J. (2000) Obesity surgery - another unmet need. British Medical Journal, 32I, 523-524.

Bateman, A. \& Fonagy, P. (1999) Effectiveness of partial hospitalisation in the treatment of borderline personality disorder: a randomised controlled trial. American Journal of Psychiatry, 156, 1563-1569.

Blackburn, R., Gunn, J., Hill, J., et al (1993) Personality disorders. In Forensic Psychiatry: Clinical, Legal and Ethical Issues (eds J. Gunn \& P. J. Taylor), pp. 373-406. Oxford: Butterworth-Heinemann.

Bourse, C. (1975) On the distinction between disease and illness. Philosophy and Public Affairs, 5, 49-68.

Campbell, E. J. M., Scadding, J. G. \& Roberts, R. S. (1979) The concept of disease. British Medical Journal, ii, 757-762.

Coccaro, E. F. \& Kavoussi, R. J. (1997) Fluoxetine and impulsive aggressive behavior in personality-disordered subjects. Archives of General Psychiatry, 54, 108|-1088.

Department of Health \& Home Office (2000) Reforming the Mental Health Act. Cm 5016. London: Stationery Office.

Drake, R. E. \& Vaillant, G. E. (1985) A validity study of Axis II of DSM-III. American Journal of Psychiatry, $\mathbf{1 4 2}$, 553-558. 
Harris, E. C. \& Barraclough, B. (1998) Excess mortality of mental disorder. British Journal of Psychiatry, I73, II-53.

\section{Home Office \& Department of Health (1999)}

Managing Dangerous People with Severe Personality

Disorder: Proposals for Policy Development. London: Home Office/Department of Health.

Kendell, R. E. (1975) The concept of disease and its implications for psychiatry. British Journal of Psychiatry, I27, 305-315.

(1986) What are mental disorders? In Issues in Psychiatric Classification (eds A. M. Freedman, R. Brotman, l. Silverman, et al), pp. 23-45. New York: Human Sciences Press.

(1993) The nature of psychiatric disorders. In Companion to Psychiatric Disorders (5th edn) (eds R. E. Kendell \& A. K. Zealley), pp. I-7. Edinburgh: Churchill Livingstone.

King, L. S. (1954) What is disease? Philosophy of Science, 2I, 193-203.
Kräupl Taylor, F. (197I) A logical analysis of the medicophysiological concept of disease. Psychological Medicine, I 356-364.

Lewis, A. (1974) Psychopathic personality: a most elusive category. Psychological Medicine, 4, 133-140.

Lewis, G. \& Appleby, L. (1988) Personality disorder: the patients psychiatrists dislike. British Journal of Psychiatry, 153, 44-49.

Lilienfeld, S. O. \& Marino, L. (1995) Mental disorder as a Roschian concept: a critique of Wakefield's 'harmfu dysfunction' analysis. Journal of Abnormal Psychology, 104 $411-420$

Linehan, M. M., Armstrong, H. E., Suarez, A., et al (199I) Cognitive-behavioral treatment of chronically parasuicidal borderline patients. Archives of General Psychiatry, 48, 1060-1064.

Martin, R. L., Cloninger, R., Guze, S B., et al (1985) Mortality in a follow up of 500 psychiatric outpatients. Archives of General Psychiatry, 42, 47-54; 58-66.
Scadding, J. G. (1967) Diagnosis: the clinician and the computer. Lancet, ii, 877-882.

Schneider, K. (1950) Die psychopatischen Personlichkeiten (9th edn). Vienna: Deuticke.

Wakefield, J. C. (1992) The concept of mental disorder: on the boundary between biological facts and social values. American Psychologist, 47, 373-388.

- (1999) Evolutionary versus prototype analyses of the concept of disorder. Journal of Abnormal Psychology, 108, 374-379.

World Health Organization (1980) International Classification of Impairments, Disabilities and Handicaps. Geneva: WHO.

- (1992a) The ICD-10 Classification of Mental and Behavioural Disorders: Clinical Descriptions and Diagnostic Guidelines. Geneva: WHO.

- (1992b) International Classification of Diseases and Related Health Problems (I0th revision) (ICD-10). Geneva: WHO. 